\title{
Participatory pattern workshops: a methodology for open learning design inquiry
}

\author{
Yishay Mor ${ }^{\mathrm{a} *}$, Steven Warburton ${ }^{\mathrm{b}}$ and Niall Winters ${ }^{\mathrm{c}}$ \\ ${ }^{a}$ Institute of Educational Technology, Open University, Milton Keynes, UK; ${ }^{b}$ International \\ Programmes, University of London, London, UK; ${ }^{c}$ Institute of Education, University of London, \\ London, UK
}

(Received 12 March 2012; final version received 17 June 2012)

\begin{abstract}
In order to promote pedagogically informed use of technology, educators need to develop an active, inquisitive, design-oriented mindset. Design Patterns have been demonstrated as powerful mediators of theory-praxis conversations yet widespread adoption by the practitioner community remains a challenge. Over several years, the authors and their colleagues have facilitated many workshops in which participants shared experiences, captured these as design narratives, extracting design patterns, and applied them to novel teaching challenges represented as design scenarios. This paper presents the core elements of the methodology that emerged from these workshops: the Participatory Patterns Workshops (PPW) methodology.
\end{abstract}

Keywords: design patterns; design narratives; scenarios; workshops; methodology

\section{Introduction}

The wealth of open and readily available information and the accelerated evolution of learning technologies offer learners and educators unprecedented opportunities, but also increasingly complex challenges. Educators are no longer learners' primary source of knowledge. Instead, they need to carefully craft the conditions for learners to inquire, explore, analyse, synthesise and collaboratively construct knowledge from the wide variety of sources available to them. This realisation is promoting a shift in the perceived role of educators, from conduits of knowledge to designers of learning experiences (Laurillard 2008). Several studies (Voogt et al. 2011) demonstrate the value of engaging in design for teachers' professional development. Extensive research over the last decade highlights the complexity of learning design and the design of learning technologies (Mor and Winters 2008). This complexity calls for novel approaches to the articulation, validation, sharing and application of design knowledge, i.e. experience of the learning design process. While design patterns can be viewed as "solutions to problems" we instead focus on their development as a way to support theory-praxis conversations (Goodyear, de Laat, and Lally 2006). This process is challenging, hence the need for our pattern workshop methodology.

This paper emerges from the work of the Learning Patterns (lp.noe-kaleidoscope. org) and Planet (patternlanguagenetwork.org) projects. Over several years, the authors and their colleagues in these projects have facilitated workshops in which

*Corresponding author. Email: Yishay.Mor@open.ac.uk 
participants shared experiences, extracted design patterns from these and applied them to novel challenges (Winters and Mor 2009). This paper presents the core elements of the methodology which emerged from these workshops: the Participatory Methodology for Practical Patterns - practical in the sense of "related to practice", and participatory in the sense that they are recorded by the practitioners from their own experience.

\section{Background}

In order to enable a culture of critical, informed and reflective design practice we need a linguistic framework for communicating design knowledge: the knowledge of the characteristic features of a domain of practice, the challenges which inhabit it and the established methods of resolving them. Several representations have been proposed to this effect: design narratives (Mor et al. 2010), design principles and design patterns (Mor and Winters 2008; Retalis, Bachfischer, and Goodyear 2010). The PPW methodology utilises two of these - design narratives and design patterns, and projects the first into the future, to form a third representation - design scenarios.

Design narratives are accounts of critical events from a personal, phenomenological perspective. They focus on design in the sense of problem solving, describing a problem in the chosen domain, the actions taken to resolve it and their unfolding effects. They provide an account of the history and evolution of a design over time, including the research context, the tools and activities designed and the results of users' interactions with these. They portray the complete path leading to an educational innovation, not just its final form - including failed attempts and the modifications they espoused. Narratives are specific by nature, posing implicit general claims by offering compelling exemplars. In order to open these general claims for critical discussion, and link them into composite structures, the design knowledge embedded in these narratives needs to be distilled in the form of design patterns. Where narratives and patterns provide a potent combination in terms of describing past design experiences, scenarios complete the picture by projecting design claims into the future. Scenarios are hypothesised narrative, articulating a design challenge in its context and proposing a possible solution for it.

Design narratives harness the power of a fundamental innate mechanism by which we organise our experiences to derive and share meanings. Bruner (1991) highlighted the epistemic force of narrative. Humans use narrative as a means of organising their experiences and making sense of them. Schank and Abelson (1975) call for a shift towards a functional view of knowledge, as Schank (1995) explains: "intelligence is really about understanding what has happened well enough to be able to predict when it may happen again" (p. 1). Such knowledge is constructed by indexing narratives of self and others' experiences, and mapping them to structures already in memory. Design narratives focus on describing a challenge of relevance to the audience and proposing a course of action for resolving it. A template and example are available at: http://www.ld-grid.org/resources/representations-andlanguages/design-narratives.

Mor and Winters (2008) provide a review of pattern approaches in educational design and research. The Design patterns paradigm (Alexander, Ishikawa, and Silverstein 1977) was developed as a form of design language within architecture. This was done with the explicit aim of externalising knowledge to allow the 
accumulation and generalisation of solutions and to allow all members of a community or design group to participate in discussions relating to design. These patterns were organised into coherent systems called pattern languages where patterns are related to each other. The original definition of a design pattern positions it as a high-level specification of a method of design which specifies the context of discussion, the particulars of the problem, and how these can be addressed by the designated design instruments. A pattern has three facets: descriptive, normative, and communicative. It is an analytic form, used to describe design situations and solutions; a meta-design tool, used to highlight key issues and dictate a method of resolving them; and a communicative tool enabling different communities to discuss design issues and solutions. The core of a design pattern can be seen as a local functional statement: "for problem $\mathrm{P}$, under circumstances $\mathrm{C}$, solution S has been known to work". Such a structure reads like a direct generalisation of the narrative form, when that narrative is a record of a problem solving effort - in other words, a design narrative. The modest nature of design patterns can also be seen as an expression of a pragmatist philosophy, suggested by several authors as the foundation of design-based research. This philosophy supports the notion of ontological innovations, which derive from the need to address the gap between practice and theory. Design patterns were described as abstractions of expert knowledge; they generalise from successful practice without detaching from its context. As such, they offer a two-way bridge between practice and theory: opening practical wisdom to theoretical scrutiny and allowing theory to be projected into practice. A template and links to some pattern collections are available at: http://www.ld-grid.org/resources/representations-and-languages/designpatterns.

Design narratives represent design knowledge extracted from empirical evidence, capturing and interpreting the designers' experience. Design patterns attempt to organise this knowledge into complex modular structures. This paper started with an identification of design knowledge. The ultimate test of any expression of design knowledge is in its success to articulate and address novel challenges in the specified domain of practice.

Design scenarios offer a suitable representation for projecting design claims into the future, posing hypothetical statements regarding potential challenges and possible solutions. They borrow the form of design narratives, adapting it from an account of documented past events to a description of imagined future ones. The context describes a current, existing situation, which is perturbed by the introduction of new material, social and intentional elements such as new technologies, new practices or new objectives. Consequently, the challenge component may describe an existing conflict of forces, which is altered by the introduction of new contextual elements. The protagonists in a design scenario do not need to refer to specific individuals in the real world, but they must describe persons who could convincingly be present in the domain of practice being explored and be ascribed with the intentions and social relations included in the described context.

At the heart of a design scenario are a sequence of actions the protagonists may take to achieve their objectives. These actions, event and consequent results are driven by the qualities of new artefacts introduced into the context. Thus, they express a design claim: that introducing such artefacts into such a context may induce such results. However, this claim is stated in a thickly grounded form, submitting it to 
elaborate scrutiny. A template and links to some examples are available at: http:// www.ld-grid.org/resources/representations-and-languages/design-scenarios.

The claim embodied in a design scenario can be judged theoretically, heuristically and empirically. Theoretical assessment would evaluate the statements in the scenario by comparing them to prior knowledge, in other words, aligning them with the relevant literature and documented case studies. Heuristic evaluation borrows from usability research, where a group of experts assess a particular design using a given rubric. Reeves et al. (2002) offer an example of applying this method to e-learning design. Finally, empirical evaluation consists of implementing the proposed design, introducing the new artefacts into the domain of practice, observing real participants reaction to them and comparing their actions to the ones in the scenario - in essence this is the standard evaluation phase of a design experiment.

\section{Workshop patterns}

The Participatory Patterns Workshops methodology is represented in its own terms; as a language of pedagogical design patterns. At the heart of the methodology is the PARTicipatory PATTERn Workshops pattern, which describes the interrelation between three Collaborative Reflection Workshops: a Design Narratives Workshop, a Design Patterns Workshop and a Design Scenarios WORKSHOP. Apart from these, the language includes a "toolkit" of support patterns, which address critical points in the process or specific recurring needs (Figure 1).

\section{Participatory Pattern WORKSHOPS}

The Participatory Methodology for Practical Design Patterns is a process by which communities of practitioners can collaboratively reflect on the challenges they face and the methods for addressing them. The outcome of the process is a set of design narratives, design patterns and future scenarios situated in a particular domain of practice. At the heart of this process are three Collaborative REFLECTION WORKSHOPS.

Collaborative Reflection WORKSHOP Elicit design knowledge by sharing, analysing and scrutinising personal experiences. This is the base structure, the "super-pattern" for all workshops.

Design Narratives

WORKSHOP

Design Patterns

WORKSHOP

Engender collaborative reflection among practitioners by a structured process of sharing stories.

Use comparative analysis of design narratives to define proto-patterns. ${ }^{1}$ Elaborate the proto-patterns to alpha-state patterns, ${ }^{2}$ by articulating the problem, context, core of the solution and related patterns.

Design SCEnARios

WORKSHOP

Put patterns to the test by applying them to novel problems in real contexts.

\section{Support toolkit patterns}

The support patterns are invoked as needed in the course of the various workshops.

DRAW AND TELL

In a conversational activity, start off by a structured task in which participants represent a personal reflection in drawing and present it to the group. The subject of the task should be related to the theme of discussion at an abstract level so that it inspires the ensuing conversation. 
PAPER 2.0

Three Hats

THIS REMINDS ME

OF ...

TABLE-TOP CONCEPT

MAPPING

Force MAPPING

Pattern Mapping

Poster Session
Paper is a wonderful technology, but Web 2.0 has some nice features. Why not combine the best of both?

I tell a story, you write it down, and she will present it.

Provoke collaborative reflection on a design narrative or scenario by asking peers to suggest similar stories.

Establish a shared vocabulary by negotiating a concept map of the problem domain.

Alexander defines a pattern as equivalent to a diagram resolving a set of interacting and conflicting forces. Many pattern authors see the articulation of forces and relations as key to the problem description. Groups of authors are asked to represent forces as icons and draw the links between them.

Groups create and compare visual maps of an emerging pattern language.

At the end of a group activity, each group produces a poster presenting its work and hangs it on the wall. Each group in turn stands before its poster and presents its work to the rest.

These patterns are described in detail in Mor, Winters, and Warburton (2010), including templates for narratives an patterns, and links to exemplar presentations. Below we provide an outline of the main patterns.

\section{Participatory pattern workshops}

The Participatory Methodology for Practical Design Patterns is a process by which communities of practitioners can collaboratively reflect on the challenges they face and the methods for addressing them. The outcome of the process is a set of design narratives, design patterns and design scenarios situated in a particular domain of practice. This pattern is an "envelope" for the rest of the patterns in this paper, and the context described here is the baseline for all the others.

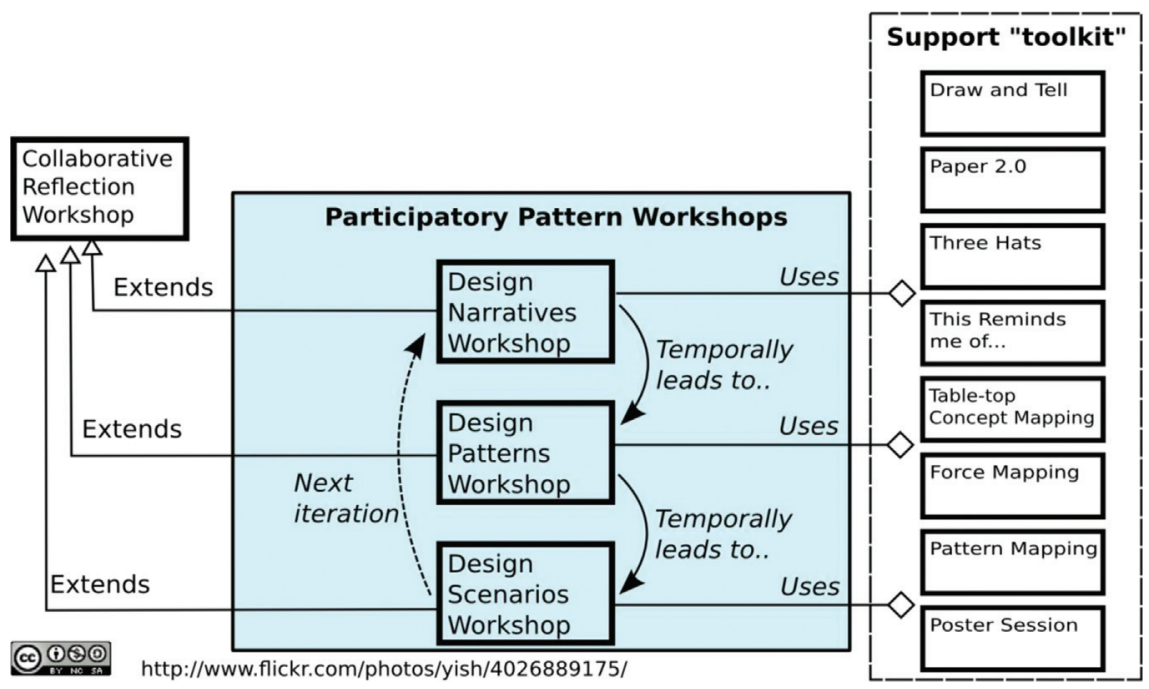

Figure 1. Map of patterns in the language. 


\section{Problem}

In order to elicit powerful and contemporary design patterns from communities of practitioners, and make these patterns useful for broad audiences, we need a structured process of guided design-level conversation, leading participants from their personal experiences to coherent pattern languages.

\section{Context}

The methodology is aimed at interdisciplinary communities of practitioners engaged in collaborative reflection on a common theme of their practice. These can be ad-hoc communities e.g. participants in a workshop, but a sense of community is nonetheless a prerequisite, in the sense of a common commitment to an inquisitive process and a genuine attempt to establish a shared discourse.

The methodology assumes a blended setting; at its heart it is a series of workshops of 4-8 hours. In between these, participants communicate and develop their ideas using an on-line collaborative authoring system. During workshops, participants refer to the on-line materials or use the system for archiving their work for later reference.

\section{Solution}

The methodology is based on two fundamental assumptions: we are all experts, and we are all designers. This methodology utilises narrative epistemology: practitioners are prompted to recount their experiences as design narratives, and discuss these with their peers. The construction and discussion of these narratives are scaffolded by a set of tools and activities to extract transferable and verifiable elements of design knowledge in the form of design patterns.

This methodology defines a process by which individuals and groups elicit structured design knowledge from their experience through a series of open yet directed activities. In an ideal setting, this process would have the following phases:

- Sharing expertise through structured stories of problems in the target domain and their resolution.

- Scrutinising and refinement of these stories by guided conversation with peers.

- Comparative analysis with respect to similar cases.

- Extraction of common features across similar cases, in terms of problem, context and method of solution.

- Grouping triplets of context, problem and solution as proto-patterns.

- Articulation of problem description by collaborative mapping of forces.

- Collaborative composition of a map of key concepts emerging from the cases and the analysis.

- Articulation of alpha-state design patterns based on the proto-patterns using the vocabulary derived from the concept mapping.

- Developing these patterns to beta-state, by providing support, in the form of triangulating cases and theoretical rationale.

- Introduction of novel problems, in the form of future scenarios.

- Validating the patterns and demonstrating their use by applying them to the scenarios. 
This process is realised by a series of COLlaborative Reflection Workshops, typically A Design Narratives Workshop, A Design Patterns Workshop and A Design Scenarios Workshop.

\section{Collaborative reflection workshop}

Elicit design knowledge by sharing, analysing and scrutinising personal experiences. This is a meta-pattern, defining the common structure for the three workshops.

\section{Problem}

Technology-infused social practices produce complex and dynamic problems. Addressing such problems requires on-going design-level conversation between designers and practitioners involved in diverse aspects of the problem domain. Such a conversation is most effective when it is grounded in actual experiences, concrete problems, relevant to the participant's current work, which have been solved or are still pending solution.

In order for such a discussion to be fruitful, it needs to be open, trusting and convivial. At the same time it should be critical, focused and output-directed. These qualities tend to create conflicting forces, in particular in ad-hoc communities, which cannot rely on established norms and relationships.

\section{Context}

This pattern assumes a co-located (on-site) half- to full-day workshop with 20-40 participants, and with a collaborative authoring system to support a-synchronous contributions before, during and after the workshop. However, it has been adapted to smaller or larger groups, and to a shorter time-frame, and to a distributed location event using technology-mediated conferencing.

\section{Solution}

Identify a theme of interest within the domain of practice. This theme should be focused enough to assume that it would draw people who can benefit from each others' experiences, and wide enough to support rich examples and dilemmas.

Convene a workshop where participants work in groups to explore the selected theme through sharing personal experience. Use a digital collaborative medium to establish rapport and set the scene before the event, by introducing the workshop methodology, asking participants to introduce themselves and share significant events. At the event, alternate between plenary sessions and group work. Each group selects a contribution of one of its members, elaborates and scrutinises it in a structured discussion. Converge to a plenary, in which each group presents its work. Conclude with a feedback and reflection discussion, in which participants recap their experience from the day. After the workshop, provide a medium for follow-up discussions and refinement of the artefacts produced on the day. 


\section{Design narratives workshop}

Engender collaborative reflection among practitioners by a structured process of sharing stories.

\section{Problem}

While everyone enjoys a good story, not everyone trusts their ability to tell a good story. People who base their confidence on a professional image often hesitate to share personal stories in public.

When people are induced to share stories, they tend to harness them to three interleaved goals: understanding the world in which they operate, establishing their identity, and identifying methods of problem solving ("where am I, who am I, how do I get where I want?"). In order to establish a productive design-level conversation, we need to subdue the first two and amplify the latter.

\section{Context}

This workshop will typically be the first in a series, followed by a DESIGN PATTERnS Workshop and a Design Scenarios Workshop. If run as a one-off event, it would be modified to include elements of the other two workshops.

\section{Solution}

Establish a case-driven discussion of common problems and solutions in the target domain, by facilitating a Collaborative Reflection Workshop, focused on participants' stories of their own experiences. The discussion is instigated by prompting participants to post their design narratives in a shared space. It culminates at a workshop, where the scenarios are analysed by groups of 3-6 participants. After the workshop, participants and facilitators revisit the cases, patterns and scenarios that were discussed.

Instruct participants to contribute a story from their own experience, using a common template. Working in groups, lead participants to examine, compare, interpret and analyse these stories using a set of guiding questions.

A template may be provided in order to guide the construction of design narratives. An example template is available at http://goo.gl/HELaC.

\section{Design patterns workshop}

Use comparative analysis of design narratives to refine candidate patterns. Elaborate the candidate patterns to full patterns, by articulating the problem, context, core of the solution and related patterns.

\section{Problem}

Design NARRATIVES WORKSHOPS guide practitioners in articulating problemsolving narratives from their experience. Narratives are a fundamental form of capturing and communicating knowledge. Yet they fall short in several accounts: 
- The endpoint of a narrative, its central message, is always implied. In order to expose it to scrutiny it needs to be made explicit.

- Narratives are loosely structured, and thus do not lend themselves to modularisation.

- Practitioners reporting on their experience often take critical factors for granted, both in terms of the context and in terms of the key actions they took.

Design patterns provide a semi-structured form which exposes the gaps and hidden messages in the design narratives, while eliminating superfluous detail. However, the transition from design narratives to patterns might seem insurmountable for the uninitiated. Many pattern communities rely on "pattern scouts", experienced pattern authors who mine practitioners' stories for potential patterns. While this approach may guarantee quality, it does not scale, and it looses the intimate knowledge of a first person account.

\section{Context}

This workshop is typically a second in a series. Ideally workshop participants should have conducted a DESIGN NARratives Workshop prior to the event, but alternatively the two workshops can be combined to one. A community dominated by experienced software designers might choose to start from this workshop, drawing on design narratives collected from other sources.

\section{Solution}

Facilitating a Collaborative Reflection Workshop which shifts the conversation from a case-driven discussion to a pattern-based discussion of common problems and solutions in the target domain. Present groups with design narratives from a previous DESIGN NARRATIVES WORKSHOP and prompt them to compare the cases and identify recurring patterns. Guide them in articulating these patterns in full.

First, ask the groups to present a short portrayal of the new pattern, by providing:

- Name

- Short description

- Illustration

Next, guide them in using a pattern template. An Exemplar template can be found at: http://goo.gl/eyZQU.

\section{Design scenarios workshop}

Put patterns to the test by applying them to novel real problems in real contexts. Guide practitioners trough a structured, quasi-scientific process of identifying an educational challenge, describing the context in which it is situated and devising a pedagogically sound solution for it. 


\section{Problem}

Design patterns provide a powerful language for such a conversation, enabling stakeholders to identify potential problems as early as possible and make an informed choice of solutions. Paradoxically, often as more expert knowledge is embedded in a pattern language it becomes less accessible to novices. In order for patterns to be used effectively by their prospective audience, they need to be presented in an approachable manner.

Furthermore, many patterns suffer from lack of validation; while they may seem compelling, this impression is not backed by unbiased empirical evidence. This reduces the audiences' confidence in patterns and creates a second obstacle to their adoption.

Such problems can be overcome by careful editing of patterns and pattern languages. Yet, with the abundance of candidate patterns which can emerge from any design discussion, for example at a Design PAtterns Workshop, we need a mechanism for prioritising efforts.

\section{Context}

Although this workshop would typically be the third in a series, following a DESIGN Narratives Workshop and a Design Patterns Workshop, alternative combinations may be more fruitful in some cases. For example, one option would be to start from scenarios and then select cases that seem to share similar problems. Alternatively, a one-off 2-day event could be organised as a Design NARRATIVES Workshop followed by a SCENARIOS WORKSHOP, leaving the patterns implicit.

\section{Solution}

Establish a scenario-driven discussion of design narratives and design patterns in a domain of practice, by facilitating a Collaborative Reflection Workshop in which participants share concrete problems in the form of future scenarios, compare them to past cases, and identify the patterns most applicable to form a solution. The discussion is instigated by prompting participants to post their scenarios in a shared space. It culminates at a workshop, where the scenarios are analysed by groups of 3-6 participants. After the workshop, participants and facilitators revisit the cases, patterns and scenarios which were discussed.

- Instruct participants to contribute a rich description of a real challenge they are confronted with in their practice, using a template, which prompts them to specify the situation, or context of the challenge and educational task - the objectives to achieve or problem to solve.

- Guide the participants in locating and reviewing relevant case studies and design narratives, and identifying appropriate design patterns.

- Describe a possible solution, based on applying the selected patterns.

- Note how the patterns themselves evolved in the process.

The template should provide additional slots for capturing these outputs, thus producing a coherent description of the problem and its proposed resolution. 
After the workshop prompt participants to publish any new design narratives, patterns and scenarios that emerged on the day, add details and artefacts (images, illustrations, diagrams, links, etc.) to their scenarios and comment on the patterns, noting questions which have emerged from the discussion.

\section{Results}

The Learning Patterns project produced around 25 design narratives and 150 patterns, 50 of them at a beta or release level of maturity. However, with the exception of Mor et al. (2010) few of them have been officially published. The pattern language network site lists over a hundred design narratives, close to 30 design patterns and 13 scenarios. This project engendered several strands of published work that included the formative e-assessment strand (Daly et al. 2010; Mor et al. 2010), which produced nine design narratives, five of which were selected for publication, and 10 patterns gathered during the JISC-funded FEASST project. Later work spread into the domain of virtual worlds and during the EC funded MUVEnation project teachers and educational researchers produced 28 design patterns, over 80 case stories and more that 20 design scenarios in the use of virtual worlds for learning and teaching (Warburton 2009). Finally, during the Rhizome project, a group of experts were brought together in the production of 11 design patterns and more than 25 case stories in the domain of digital identity management (Warburton 2010).

Recently, the methodology has been used with promising results by the ML4D project in the domain of mobile learning for development.

\section{Discussion}

Our account is based on scores of workshops conducted in a wide range of settings and targeting a variety of audiences. Nevertheless, the question of scalability and extendibility needs to be considered. How robust is the methodology described here to the constraints of particular situations? How much does it rely on our personal qualities as facilitators? When would it be effective, and when would be an overkill?

Our experience spans instances where one, two or three of us have been involved in facilitating workshops, often in collaboration with other colleagues. These ranged from one-off, half-day events, to series of three to five full-day workshops, and with between 20 and 40 participants in each event. As this is the first publication of this methodology, we do not yet have independent accounts from others who have tried it, which would lend it further credibility.

This broad base of evidence would suggest that the methodology is robust and versatile. Although it has presented here as a monolith, it is in fact modular - and has been successfully adapted, e.g. by running a stand-alone scenarios workshop, or a combined narratives and patterns workshop.

Participatory workshops are probably not the most cost-effective means of collecting and disseminating design knowledge. Any participatory process by definition is a time-intensive activity. On the other hand, the workshops offer participants significant personal benefits, in terms of their professional development, and they produce outputs which reflect the social and individual knowledge assets of participants.

This methodology is not "a tool for all trades". Mor (forthcoming) describes a related methodology which is more suitable for individuals or teams conducting 
extensive research or development projects. The participatory pattern methodology is most effective in eliciting the "design Zeitgeist" of a professional community, while enhancing the design discourse within that community.

\section{Conclusions}

The methodology presented above is a product of our collaborative reflection on scores of workshops conducted over several years. These workshops ranged from one-off events of several hours to series of three to five encounters, each one up to a full day. Each event or series was dedicated to a particular theme, and its primary outputs were relevant to the participants interested in that theme. These outputs included design narratives, design patterns and design scenarios of varied maturity. They also included the intangible experience of the participants and the insights they derived from them, allowing them to perceive their domain with a new perspective: a design view which transcends the clutter of daily detail, but is still readily applicable for them and their peers. It is here that the power of design patterns becomes visible via their non-prescriptive nature, which allows experts to share their knowledge without imposing a singular method of solution.

The methodology has since been used by several groups with notable success including FEASST (feedback and assessment), MUVENation (immersive virtual worlds), Rhizome (digital identity) and the ML4D (learning design) projects. Naturally, each instance presented its unique conditions and constraints, and thus, in true Alexandrian fashion, each of these patterns "describes a problem which occurs over and over again in our environment, and then describes the core of the solution to that problem, in such a way that you can use this solution a million times over, without ever doing it the same way twice" (Alexander, Ishikawa, and Silverstein 1977).

\section{Acknowledgements}

The Pattern Language Network (Planet) project was funded by JISC. The Learning Patterns was a Jointly Executed Integrating Research Project of the Kaleidoscope Network of Excellence. Additional support via Eduserv funded Rhizome project and JISC funded formative e-assessment project.

\section{Notes}

1. Proto-patterns represent the first iteration of a pattern that captures the basic elements of the problem, solution and context.

2. Alpha-state denotes patterns that have undergone refinement through a number of iterations to a state where they can be released for general use and testing by designers.

\section{References}

Alexander, C., Ishikawa, S. \& Silverstein, M. (1977) A Pattern Language: Towns, Buildings, Construction, Oxford University Press, New York, NY.

Bruner, J. (1991) 'The Narrative Construction of Reality', Critical Inquiry, vol. 18. Harvard Lectures, Harvard University Press, Cambridge, MA. pp. 1-21. Available at: http://www. semiootika.ee/sygiskool/tekstid/bruner.pdf.

Daly, C., et al. (2010) 'Exploring formative e-assessment: using case stories and design patterns', Assessment \& Evaluation in Higher Education, vol. 35, no. 5, pp. 619-636.

Goodyear, P., de Laat, M. \& Lally, V. (2006) 'Using pattern languages to mediate theory-praxis conversations in design for networked learning', $A L T-J$, vol. 14, no. 3, pp. 211-223. 
Laurillard, D. (2008) 'The teacher as action researcher: using technology to capture pedagogic form', Studies in Higher Education, vol. 33, no. 2, pp. 139-154.

Mor, Y. (forthcoming) 'SNaP! Re-using, sharing and communicating designs and design knowledge using Scenarios, Narratives and Patterns', in Handbook of Design in Educational Technology, eds R. Luckin, P. Goodyear, B. Grabowski, S. Puntambekar, N. Winters \& J. Underwood, Routledge.

Mor, Y., et al. (2010) 'Formative e-Assessment: case stories, design patterns and future scenarios', in Problems Investigations of E-Learning Patterns: Context Factors Solutions, eds C. Kohls \& J. Wedekind, Information Science Publishing, Hershey, PA, pp. 199-219.

Mor, Y. \& Winters, N. (2008) 'Participatory design in open education: a workshop model for developing a pattern language', Journal of Interactive Media, Special Issue. Available at: http://www-jime.open.ac.uk/article/2008-13/350

Mor, Y., Winters, N. \& Warburton, S. (2010) Participating Pattern Workshops: Resource Kit. Available at: www.warburton.typepad.com/files/ppw_2_1.pdf

Retalis, S., Bachfischer, A. \& Goodyear, P. (2010) 'Design patterns for technology-enhanced learning: achievments and opportunities', in Technology-Enhanced Learning: Design Patterns and Pattern Languages, eds S. Retalis \& P. Goodyear, Sense, Rotterdam, pp. 311-318.

Reeves, T. C., et al. (2002) 'Usability and instructional design heuristics for e-learning evaluation', in Proceedings of World Conference on Educational Multimedia, Hypermedia and Telecommunications, eds P. Barker \& S. Rebelsky, AACE, Chesapeake, VA, pp. $1615-1621$.

Schank, R. (1995) Tell Me a Story: Narrative and Intelligence, Northwestern University Press, Evanston, IL.

Schank, R. C. \& Abelson, R. P. (1975) 'Scripts, plans and knowledge', in Thinking: Readings in Cognitive Science, Proceedings of the Fourth International Joint Conference on Artificial Intelligence, eds P. N. Johnson-Laird \& P. C. Wason, USSR, Tbilisi, pp. 151-157.

Voogt, J. et al. (2011) 'Teacher learning in collaborative curriculum design', Teaching and Teacher Education, vol. 27, no. 8, pp. 1235-1244.

Warburton, S. (2009) 'WP4c Report on Design Patterns for Virtual Worlds', Available at: http://dl.dropbox.com/u/4424450/KCL_v6_MUVENATIONDesignPatternsWP4.pdf

Warburton, S. ed. (2010) Digital Identity Matters. King's College London, London. Available at: http://digitaldisruptions.org/rhizome/wp-content/uploads/2010/06/rhiz08_ DigitalIdentityMatters.pdf

Winters, N. \& Mor, Y. (2009) 'Dealing with abstraction: case study generalisation as a method for eliciting design patterns', Computers in Human Behavior, vol. 25, no. 5, pp. 1079-1088. 